\title{
Influência da adição de erva-mate (Ilex paraguariensis A. St. Hil.) em pó nas características físico-químicas e no potencial bioativo de hidroméis
}

\author{
Influence of the addition of yerba mate (Ilex paraguariensis A. St. Hil.) in powder on the \\ physicochemical characteristics and bioactive potential of meads \\ Influencia de la adición de yerba mate (Ilex paraguariensis A. St. Hil.) en polvo sobre las \\ características fisicoquímicas y bioactivo potencial del hidromiel
}

Recebido: 02/07/2021 | Revisado: 14/07/2021 | Aceito: 16/07/2021 | Publicado: 25/07/2021

\author{
Monnik Gandin Cavanholi \\ ORCID: https://orcid.org/0000-0001-6426-6690 \\ Universidade Federal de Santa Catarina, Brasil \\ E-mail: nikgandin@gmail.com \\ Bruna Rafaela da Silva Monteiro Wanderley \\ ORCID: https://orcid.org/0000-0001-7075-1086 \\ Universidade Federal de Santa Catarina, Brasil \\ E-mail: brunarafaelawanderley@gmail.com \\ Gabriela Soster Santetti \\ ORCID: https://orcid.org/0000-0002-5382-9264 \\ Universidade Federal de Santa Catarina, Brasil \\ E-mail: zz_gabriela@hotmail.com \\ Renata Dias de Mello Castanho Amboni \\ ORCID: https://orcid.org/0000-0002-6467-3760 \\ Universidade Federal de Santa Catarina, Brasil \\ E-mail: renata.amboni @ufsc.br \\ Carlise Beddin Fritzen-Freire \\ ORCID: https://orcid.org/0000-0002-6248-0914 \\ Universidade Federal de Santa Catarina, Brasil \\ E-mail: carlise.freire@ufsc.br
}

\begin{abstract}
Resumo
O hidromel é uma bebida alcoólica, resultante da fermentação de mel diluído em água, na presença de leveduras. Uma forma de diversificação da bebida é a adição de frutas e ervas. A erva-mate (Ilex paraguariensis A. St. Hil.) é uma planta nativa da América do Sul, sendo amplamente estudada por ser uma excelente fonte de compostos fenólicos e com elevada capacidade antioxidante. Assim, o objetivo deste trabalho foi avaliar a influência da adição de erva-mate em pó nas propriedades físico-químicas e no potencial bioativo de hidroméis. Para isso, foram elaboradas três formulações de hidromel: (HC): controle - sem adição de erva-mate; (HF): com adição de $1 \%$ de folhas de erva-mate em pó submetidas à extração aquosa à frio; e (HQ): com adição de $1 \%$ de folhas de erva-mate em pó submetidas à extração aquosa à quente. Avaliou-se os mostos e as amostras de hidromel quanto aos parâmetros físico-químicos, de cor, teor de compostos fenólicos totais e capacidade antioxidante in vitro. A adição dos extratos de erva-mate contribuiu para o aumento da acidez dos mostos e dos hidroméis. Todas as amostras tiveram uma queda significativa do teor de sólidos solúveis totais após a fermentação, e consequentemente um aumento do teor alcoólico. Os valores obtidos para o teor de compostos fenólicos totais e para a capacidade antioxidante foram mais elevados para as amostras com extrato de erva-mate, tanto nos mostos quanto nos hidroméis. Assim, os resultados revelaram que a erva-mate apresenta-se como uma alternativa para aumentar o potencial bioativo do hidromel.
\end{abstract}

Palavras-chave: Mel; Bebidas alcoólicas; Fermentação; Capacidade antioxidante; Compostos fenólicos totais.

\begin{abstract}
Mead is an alcoholic beverage, resulting from the fermentation of honey dilution in water, in the presence of yeast. The diversification of mead can be carried by out addition of fruits and herbs. Yerba mate is a native plant of South America, being widely studied for being an excellent source of phenolic compounds and with high antioxidant capacity. Thus, the objective of this work was to evaluate the influence of addition of yerba mate powder on the physicochemical properties and on the bioactive potential of meads. For this, three formulations of mead were elaborated: (HC): control - without addition of yerba mate; (HF): with the addition of $1 \%$ yerba mate in powder leaves submitted to cold aqueous extraction; and (HQ): with the addition of $1 \%$ yerba mate in powder leaves submitted to hot aqueous extraction. Musts and meads were evaluated for physical parameters-compounds, color, content of total phenolic compounds, in vitro antioxidant capacity, and the alcohol content of the meads. The addition of yerba mate
\end{abstract}


extracts contributed to increase the acidity of musts and meads. When analyzing the total solids, all after the reduction there is a decrease in fermentation, and an increase in alcohol content. The values regarding the obtainment for the content of total phenolic compounds and for the antioxidant capacity were the highest for those with yerba mate extract, both in the musts in the meads. Thus, the results obtained in this study revealed that mate is an alternative to increase the bioactive potential of mead.

Keywords: Honey; Alcoholic beverage; Fermentation; Antioxidant capacity; Total polyphenols.

\section{Resumen}

El hidromiel es una bebida alcohólica, resultante de la fermentación de la miel diluida en agua, en presencia de levadura, se puede agregar hierbas y frutas. La yerba mate es una planta nativa de América del Sur, siendo ampliamente estudiada por ser una excelente fuente de compuestos fenólicos y con alta capacidad antioxidante. El objetivo de este trabajo fue evaluar la influencia de la adición de polvo de yerba mate sobre las propiedades fisicoquímicas y el potencial bioactivo de lo hidromiel. Para ello, se elaboraron tres formulaciones de hidromiel: (HC): control - sin adición de yerba mate; (HF): con la adición de 1\% de yerba mate en polvo, hojas sometidas a extracción acuosa fría; y (HQ): con la adición de 1\% de hojas de yerba mate en polvo sometidas a extracción acuosa en caliente. Las muestras de mostos y hidromiel se evaluaron en cuanto a parámetros físicoquímicos, color, contenido de compuestos fenólicos totales y capacidad antioxidante in vitro. La adición de extractos de yerba mate contribuyó a incrementar la acidez de mostos e hidromiel. Todas las muestras tuvieron una caída significativa de sólidos solubles totales después de la fermentación y un aumento en el contenido de alcohol. Los valores obtenidos para el contenido de compuestos fenólicos totales y para la capacidad antioxidante fueron mayores para las muestras con extracto de yerba mate, tanto en mostos como en hidromiel. Así, los resultados obtenidos revelaron que la yerba mate se presenta como una alternativa para incrementar el potencial bioactivo del hidromiel.

Palabras clave: Miel; Bebida alcohólica; Fermentación; Capacidad antioxidante; Polifenoles totales.

\section{Introdução}

O hidromel é uma bebida alcoólica fermentada a partir de mosto de mel apropriadamente diluído (Mendes-Ferreira et al., 2010). Esta bebida tem um histórico milenar e mesmo na atualidade é apreciada pelos consumidores, sendo que o seu consumo vem aumentando nos últimos anos (Iglesias et al., 2014). A qualidade da bebida e a sua composição química estão relacionadas a diferentes fatores, como o tipo de mel, aditivos, cepa de levedura empregada, bem como a composição do mosto e as etapas e condições do processo fermentativo (Akalin et al., 2017).

Para a elaboração de hidromel, as leveduras do gênero Saccharomyces são comumente utilizadas pela sua alta capacidade de conversão dos açúcares em etanol (Mendes-Ferreira et al., 2010). Starowicz \& Granvogl, (2020) relatam ainda que a produção de etanol em hidroméis está relacionada especialmente com a proporção de mel empregada na elaboração do mosto. Além disso, o hidromel apresenta grande potencial para adição de diferentes ingredientes, como cereais, ervas ou frutas (Kawa-Rygielska et al., 2019) que podem promover a valorização do produto, além de influenciar as características dessa bebida, principalmente devido à incorporação de compostos bioativos.

A erva-mate (Ilex paraguariensis A. St. Hil.) é uma planta nativa da América do Sul, muito apreciada no Uruguai, Argentina, Brasil e Paraguai, sendo tradicionalmente empregada para o preparo do chimarrão, por infusão em água quente de suas folhas secas e picadas, e do tererê, que utiliza infusão à frio (Thea et al., 2016). As suas folhas apresentam uma composição rica em compostos fenólicos (Boaventura et al., 2015; Santos et al., 2017). No entanto, as concentrações destes compostos estão diretamente relacionadas ao tipo de extração empregada (Correa et al., 2017). Devido ao crescente interesse dos consumidores por alimentos com potencial bioativo, o desenvolvimento de hidromel contendo erva-mate em pó pode ser uma opção inovadora de valorização de matérias-primas regionais, levando à diversificação da bebida.

Diante disto, o objetivo deste estudo foi avaliar a influência da adição de erva-mate em pó nas propriedades físicoquímicas e no potencial bioativo de hidroméis.

\section{Metodologia}

O estudo corresponde a uma pesquisa laboratorial aplicada de natureza experimental e abordagem quantitativa, 
mediante coleta de dados numéricos e análise estatística (Pereira et al., 2018).

\subsection{Amostras}

As folhas de erva-mate (Ilex paraguariensis A. St. Hil) in natura foram obtidas da safra 2017. As folhas foram lavadas manualmente em água corrente para retirada das sujidades aparentes e posteriormente submetidas ao processo de inativação enzimática, onde foram submersas em água a $95 \pm 2{ }^{\circ} \mathrm{C}$ durante 30 segundos, e em seguida resfriadas por imersão em água contendo gelo. Após esta etapa, as folhas passaram pelo processo de pré-secagem em centrífuga manual para retirada do excesso de água, e em seguida, foram secas em estufa com circulação de ar a $45 \pm 2{ }^{\circ} \mathrm{C}$ durante 24 horas. As folhas secas foram submetidas a moagem em moinho de facas (Marconi, M048, São Paulo, Brasil), até atingir os tamanhos de partículas na faixa de $\leq 245 \mu \mathrm{m}$. As amostras foram identificadas e acondicionadas em embalagens de polietileno metalizadas e levadas para o armazenamento em temperatura de $-18 \pm 2{ }^{\circ} \mathrm{C}$.

\subsection{Processo de fermentação}

Os mostos foram elaborados de acordo com a metodologia proposta por Roldán et al. (2011), com modificações, utilizando mel comercial silvestre (Apiário Real, São Joaquim, Brasil) (Apis mellifera), com cerca de $80{ }^{\circ}$ Brix e água mineral (Imperatriz, Santo Amaro da Imperatriz, Brasil). Foram elaboradas três formulações de hidromel: (HC): hidromel controle (o mel foi diluído em água mineral a $30^{\circ} \mathrm{C}$ até obter um mosto com cerca de $22^{\circ}$ Brix); (HF): hidromel com erva-mate com extração à frio (diluiu-se o mel em água mineral a $30^{\circ} \mathrm{C}$ contendo $1 \%$ de erva-mate em pó $(\mathrm{m} / \mathrm{m})$ até obter um mosto com cerca de $22^{\circ} \mathrm{Brix}$ ); e (HQ): hidromel com erva-mate com extração à quente (diluiu-se o mel em um extrato aquoso a $30^{\circ} \mathrm{C}$ contendo $1 \%$ de erva-mate em pó $(\mathrm{m} / \mathrm{m})$ até obter um mosto com cerca de $22^{\circ}$ Brix). Para a extração à quente, a mistura de água e erva-mate em pó foi submetida à $90{ }^{\circ} \mathrm{C}$ por 5 minutos, com posterior resfriamento a $30^{\circ} \mathrm{C}$. Em todas as formulações foram adicionados metabissulfito de potássio $(50 \mathrm{mg} / \mathrm{L})$ e cultura comercial de Saccharomyces bayanus (1g/L) (Red Star Premier Blanc, Langerbruggekaai, Bélgica). O processo fermentativo foi realizado em fermentadores de vidro, em incubadora BOD (TE-371, Tecnal, Piracicaba, Brasil) a $25^{\circ} \mathrm{C}$ e acompanhado rotineiramente, durante 21 dias, através de medidas de sólidos solúveis totais, $\mathrm{pH}$ e pesagem dos fermentadores (até peso constante). Ao final da fermentação os hidroméis foram refrigerados $\left(5^{\circ} \mathrm{C}\right)$ por uma semana e na sequência foram filtrados e engarrafados.

\subsection{Análises físico-químicas e de cor}

As análises físico-químicas foram realizadas nos mostos e nos hidroméis. A determinação do $\mathrm{pH}$ foi realizada em potenciômetro (Tec-7, Tecnal, Piracicaba, Brasil), a acidez total (\% ácido cítrico) foi determinada de acordo com as metodologias descritas no Manual de Normas Analíticas do Instituto Adolfo Lutz (IAL, 2008). O teor de sólidos solúveis totais ( ${ }^{\circ}$ Brix) foi determinado em refratômetro digital (HI96801, Hanna Instruments, Barueri, Brasil). O teor alcoólico dos hidroméis foi determinado em triplicata, de acordo com a metodologia proposta pela Organização Internacional da Vinha e do Vinho (OIV, 2012).

A análise da cor dos mostos e dos hidroméis foi determinada através de um colorímetro (Minolta Chroma Meter CR400, Osaka, Japão), ajustado para operar com iluminante D65 e ângulo de observação de $10^{\circ}$, previamente calibrado. A escala de cor CIELab foi usada para medir os parâmetros $L^{*}, a^{*}, b^{*}$. Na escala CIELab, o parâmetro $L^{*}$ varia de 0 a 100, indicando

uma variação de cor do preto ao branco. Já o parâmetro $a^{*}$ mostra a variação do vermelho $\left(+a^{*}\right)$ ao verde (-a*), enquanto o $b^{*}$ pode variar do amarelo $\left(+b^{*}\right)$ ao azul (-b*). Todas as análises foram realizadas em triplicata. 


\subsection{Teor de compostos fenólicos totais e capacidade antioxidante in vitro}

$\mathrm{O}$ teor de compostos fenólicos totais e a capacidade antioxidante in vitro foram determinados nos mostos e nos hidroméis. O teor de polifenóis totais foi determinado pelo método de Folin-Ciocalteu (Singleton \& Rossi, 1965). A leitura da absorbância foi realizada a $765 \mathrm{~nm}$ em espectrofotômetro UV-VIS (modelo U-1800, Hitachi, Japão) e os resultados foram expressos em mg equivalente de ácido gálico (EAG) por litro (L) de amostra. A capacidade antioxidante foi determinada pelos métodos de atividade de eliminação de radicais livres ABTS+ (Re et al., 1999) e pelo potencial antioxidante redutor férrico (FRAP) (Benzie \& Strain, 1996). Os resultados dos ensaios de ABTS e FRAP foram expressos em $\mu$ M equivalente de Trolox (TEAC) por litro (L) de amostra.

\subsection{Análise estatística}

As análises de variância (ANOVA), teste de Tukey $(\mathrm{p}<0,05)$, análise de correlação e análise dos componentes principais (ACP) foram realizadas no software STATISTICA versão 13.3 (StatSoft Inc., Tulsa, OK, E.U.A.). Todas as análises foram realizadas em triplicata e os resultados expressos em média \pm desvio padrão.

\section{Resultados e Discussão}

\subsection{Avaliação da fermentação dos hidroméis}

O processo fermentativo dos hidroméis teve duração de 21 dias e ao longo deste período foram realizadas análises periódicas do teor de sólidos solúveis totais (SST) e do $\mathrm{pH}$ para verificação de consumo do substrato pelas leveduras e da atividade fermentativa (Figura 1).

Figura 1 - Evolução do teor de sólidos solúveis totais (A) e do pH (B) durante o processo de fermentação dos hidroméis.
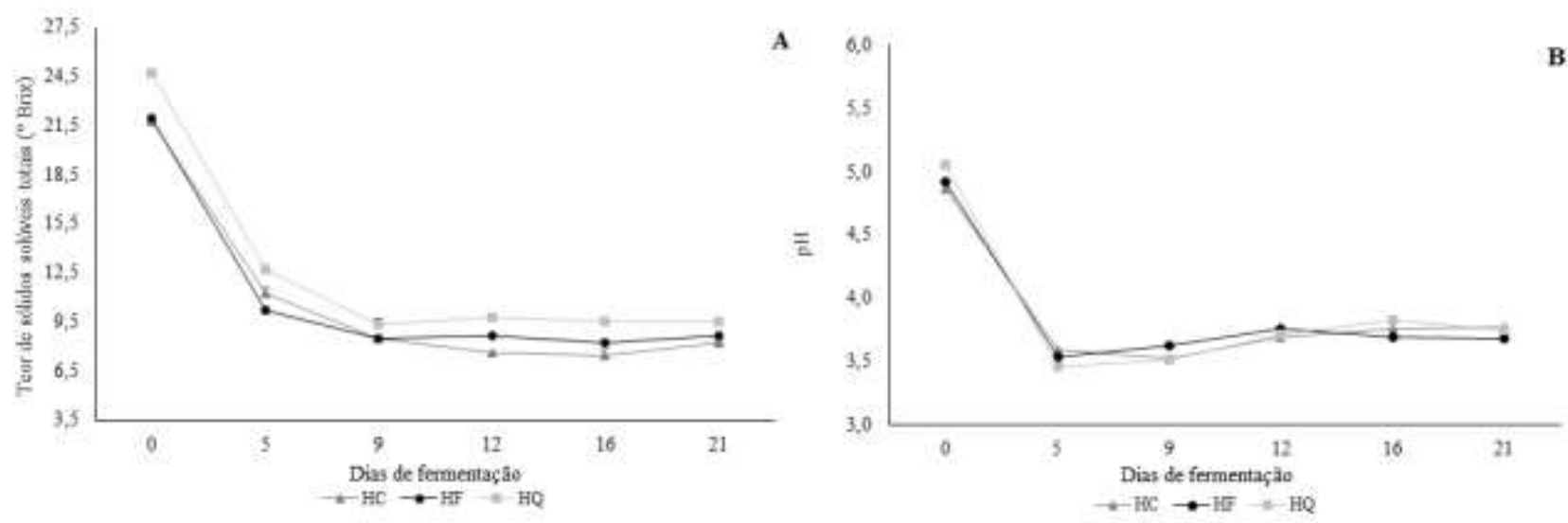

Fonte: Autores (2021). HC: hidromel controle; HF: hidromel com erva-mate com extração à frio; HQ: hidromel com erva-mate com extração à quente.

Nos primeiros nove dias de fermentação houve uma grande diminuição $(p<0,05)$ nos valores de sólidos solúveis totais em todas as amostras analisadas, demonstrando um grande potencial na transformação de açúcares em etanol pelas leveduras (Figura 1 - A). Do nono dia até o vigésimo primeiro dia, o processo de fermentação seguiu com um comportamento mais lento, com menor metabolização dos açúcares dos mostos, até os fermentadores apresentarem peso constante.

A figura 1 (B) apresenta uma queda elevada $(\mathrm{p}<0,05)$ e constante dos valores de $\mathrm{pH}$ para todas as amostras de hidromel até o quinto dia de fermentação. Comportamento semelhante foi observado por Chen et al. (2013), que avaliaram 
modificações físico-químicas durante a fermentação de hidroméis elaborados com diferentes leveduras. Após o quinto dia de fermentação observou-se uma estabilidade do pH das amostras ( $\mathrm{p}>0,05$ ). De acordo com Pereira et al. (2009), além do etanol, outros compostos são gerados durante a fermentação do hidromel, incluindo os ácidos orgânicos, que podem alterar o pH da bebida. Sroka \& Tuszyński, (2007) demostraram que os ácidos acéticos e succínico formados durante a fermentação do hidromel diminuíram o pH da bebida, promovendo a desaceleração do processo fermentativo das bebidas avaliadas.

\subsection{Avaliação das propriedades físico-químicas dos mostos e dos hidroméis}

A avaliação físico-química dos mostos (antes do processo fermentativo) e dos hidroméis (após o processo fermentativo) foi realizada a partir das análises de sólidos solúveis totais (SST), pH e acidez e os resultados estão apresentados na Tabela 1.

Tabela 1 - Valores médios \pm desvio padrão dos parâmetros físico-químicos das amostras de mosto e de hidromel: controle (MC e HC), com erva-mate com extração à frio (MF e HF) e com erva-mate com extração à quente (MQ e HQ).

\begin{tabular}{cccc}
\hline Tratamentos & SST $\left({ }^{\circ}\right.$ Brix $)$ & $\mathbf{p H}$ & Acidez $(\mathbf{m E q} / \mathbf{L})$ \\
\hline MC & $21,90 \pm 0,10^{\mathrm{aA}}$ & $4,86 \pm 0,06^{\mathrm{aA}}$ & $5,50 \pm 0,50^{\mathrm{aA}}$ \\
MF & $21,93 \pm 0,06^{\mathrm{aA}}$ & $4,92 \pm 0,02^{\mathrm{aA}}$ & $14,50 \pm 0,50^{\mathrm{bA}}$ \\
MQ & $24,73 \pm 0,06^{\mathrm{bA}}$ & $4,99 \pm 0,04^{\mathrm{aA}}$ & $15,50 \pm 0,50^{\mathrm{bA}}$ \\
& & & \\
HC & $8,30 \pm 0,17^{\mathrm{aB}}$ & $3,85 \pm 0,02^{\mathrm{abB}}$ & $49,50 \pm 1,50^{\mathrm{aB}}$ \\
HF & $8,83 \pm 0,06^{\mathrm{bB}}$ & $3,69 \pm 0,03^{\mathrm{bB}}$ & $60,50 \pm 1,50^{\mathrm{bB}}$ \\
HQ & $9,90 \pm 0,26^{\mathrm{cB}}$ & $3,82 \pm 0,03^{\mathrm{aB}}$ & $60,00 \pm 0,00^{\mathrm{bB}}$
\end{tabular}

Fonte: Autores (2021). ${ }^{\mathrm{a}-\mathrm{c}}$ Diferentes letras minúsculas sobrescritas na mesma coluna indicam diferenças significativas ( $\left.<<0,05\right)$ entre as amostras para a mesma categoria de amostra (mosto ou hidromel). ${ }^{\text {A-B }}$ Diferentes letras maiúsculas sobrescritas na mesma coluna indicam diferenças significativas $(\mathrm{p}<0,05)$ entre os tratamentos para a mesma amostra.

Os resultados de sólidos solúveis totais obtidos para os mostos demonstram que não houve uma diferença $(p>0,05)$ entre as amostras controle (MC) e com erva-mate com extração à frio (MF). No entanto, o mosto com erva-mate com extração à quente (MQ) apresentou maior ( $<$ 0,05) valor de SST do que as demais amostras. Gomes et al. (2013), ao avaliarem o efeito do tratamento térmico na elaboração de hidroméis perceberam que a utilização de temperaturas elevadas na elaboração das bebidas resultou em um aumento da concentração de açúcares nos mostos. No entanto, neste trabalho, apenas a erva-mate foi submetida ao tratamento térmico, sendo o mel adicionado após o resfriamento do extrato aquoso, indicando uma influência da temperatura e do tempo de extração da erva-mate na concentração dos sólidos solúveis deste mosto. Ao comparar os resultados de sólidos solúveis totais após a fermentação dos hidroméis verificaram-se diferenças $(\mathrm{p}<0,05)$ entre as três amostras, sendo que o hidromel controle (HC) apresentou os menores valores de SST. No entanto, comparando os dados dos mostos e dos hidroméis analisados, observou-se uma grande diminuição $(\mathrm{p}<0,05)$ do teor de SST em todas as amostras durante a fermentação, indicando que houve uma conversão eficiente de açúcares em etanol pelas leveduras.

Quanto aos resultados de $\mathrm{pH}$, não foram observadas diferenças $(\mathrm{p}>0,05)$ entre os mostos, com valores variando entre 4,86 e 4,99. De acordo com Lin et al. (2012), a capacidade de produção de etanol está diretamente relacionada ao pH do 
mosto. Estes autores consideraram uma faixa ótima de produção de etanol entre pH 4,0 e 5,0, sendo que valores inferiores a 4,0 e superiores a 5,0 tornam o processo mais lento, exigindo maior tempo de incubação para que se possa atingir uma maior concentração do etanol no sistema. Após a fermentação, foi verificada uma diminuição nesse parâmetro $(p<0,05)$ para todas as amostras, sendo que o hidromel controle não apresentou diferença (p > 0,05) em relação à amostra HF e HQ. KawaRygielska et al. (2019) avaliaram hidroméis contendo ervas e frutas e observaram diminuição do pH das amostras com o avanço da fermentação. De acordo com estes autores, a diminuição do pH se deve ao processo fermentativo, pois durante esse processo são gerados ácidos orgânicos através do metabolismo das leveduras, que contribuem para a diminuição deste parâmetro. Ao avaliar os resultados de acidez, percebeu-se uma diferença expressiva $(\mathrm{p}<0,05)$ entre o mosto controle e os mostos com extrato de erva mate (MF e MQ), que apresentaram uma acidez mais elevada. Com isto, os resultados sugerem que a adição de $1 \%$ de erva-mate em pó nos mostos de hidromel, independentemente do tratamento térmico, contribuiu para o aumento da acidez nas amostras. Comportamento semelhante foi observado após a fermentação, com um aumentou considerável $(\mathrm{p}<0,05)$ da acidez total nas amostras de hidromel, sendo mais significativo nas amostras com erva-mate. Sroka \& Tuszyński, (2007) afirmaram que o aumento da acidez total é ocasionado pela síntese de ácidos orgânicos durante a fermentação do hidromel e como consequência o pH do sistema tende a diminuir.

O etanol é o metabólito primário que se deseja na fermentação alcoólica, neste sentido, os resultados obtidos para teor alcoólico nos hidroméis HC, HF e HQ foram: 10,10 $\pm 0,01 \%, 10,05 \pm 0,00 \%$ e 11,05 \pm ,00\%, respectivamente. Estes resultados indicaram diferenças $(\mathrm{p}<0,05)$ entre as amostras de hidroméis, com um maior teor alcoólico para o hidromel elaborado com erva-mate com extração à quente. Este resultado provavelmente ocorreu devido ao maior teor de SST iniciais desta amostra (mosto antes da fermentação - Tabela 1) decorrente da concentração de açúcares durante a extração à quente, favorecendo uma maior produção de etanol pelas leveduras. Além disso, os valores obtidos para ambas as amostra estão de acordo com o relatado por Starowicz \& Granvogl, (2020), onde hidroméis apresentam um teor alcoólico entre 8-18\%.

Os resultados dos parâmetros de cor $\mathrm{L}^{*}, \mathrm{a}^{*} \mathrm{e} \mathrm{b}^{*}$ dos mostos (antes do processo fermentativo) e dos hidroméis (após o processo fermentativo) estão apresentados na Figura 2.

Figura 2 - Valores médios \pm desvio padrão dos parâmetros de cor das amostras de mosto e de hidromel: controle (MC e HC), com erva-mate com extração à frio (MF e HF) e com erva-mate com extração à quente (MQ e HQ).

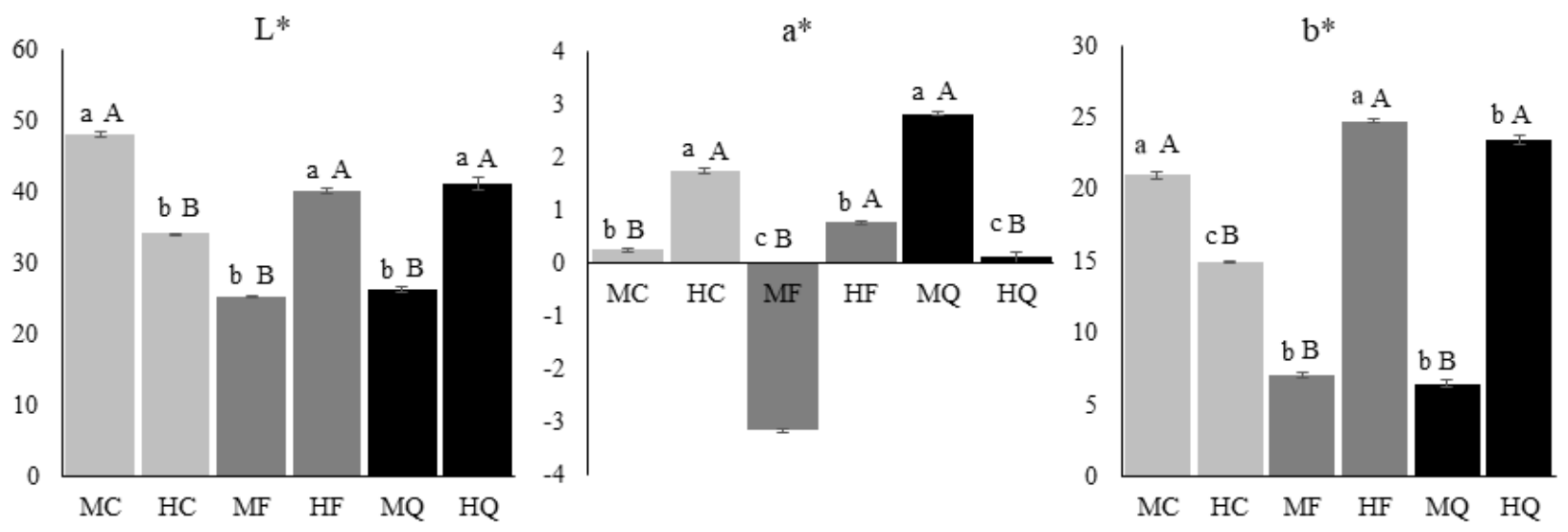

Fonte: Autores (2021). ${ }^{\mathrm{a}-\mathrm{c}}$ Diferentes letras minúsculas sobrescritas na mesma coluna indicam diferenças significativas $(\mathrm{p}<0,05)$ entre as amostras para a mesma categoria de amostra (mosto ou hidromel). ${ }^{\text {A-B }}$ Diferentes letras maiúsculas sobrescritas na mesma coluna indicam diferenças significativas $(\mathrm{p}<0,05)$ entre os tratamentos para a mesma amostra. 
As amostras de mosto e de hidromel apresentaram baixa luminosidade ( $\left.\mathrm{L}^{*} \leq 50\right)$, devido à influência das matériasprimas empregadas. De acordo com Saxena et al. (2010), quando o mel apresenta um L*> 50 é caracterizado como um mel claro, enquanto os méis mais escuros possuem um $\mathrm{L}^{*} \leq 50$. No entanto, antes da fermentação, os mostos dos hidroméis contendo os extratos de erva-mate (MF e MQ) apresentaram menor ( $<$ < 0,05) luminosidade em relação ao mosto controle, indicando que a utilização de $1 \%$ de erva-mate em pó exerceu influência neste parâmetro. Por outro lado, após a fermentação, a luminosidade diminuiu $(\mathrm{p}<0,05)$ no hidromel controle e aumentou $(\mathrm{p}<0,05)$ nos hidroméis com erva-mate. Este aumento na luminosidade pode ser decorrente do processo de filtração que as amostras foram submetidas.

O parâmetro a* indica a variação do verde (-) ao vermelho (+) (Saxena et al., 2010). Assim, observou-se que os mostos diferiram entre si $(\mathrm{p}<0,05)$ neste parâmetro, com as amostras MC e MQ apresentando uma tendência à cor vermelha, enquanto a amostra MF apresentou uma tendência à cor verde. Este comportamento pode estar relacionado à preservação dos pigmentos verdes da erva-mate na amostra que não foi submetida à extração à quente. De acordo com Andrés-Bello et al. (2013), a estabilidade da clorofila é comprometida quando submetida a uma temperatura superior a $50^{\circ} \mathrm{C}$, alterando a coloração do alimento. Após a fermentação, observou-se que todas as amostras apresentaram tendência ao vermelho, com diferentes intensidades ( $\mathrm{p}<0,05)$. De acordo com Schmalko et al. (2005), quando a clorofila sofre degradação, a coordenada a*é afetada, apresentando tendência ao vermelho. No entanto, a amostra HQ apresentou diminuição do valor de a* após a fermentação, provavelmente devido ao processo de filtração da bebida.

Quanto aos resultados do parâmetro $b^{*}$, todos os mostos e hidroméis apresentaram uma tendência à cor amarela, com maiores $(\mathrm{p}<0,05)$ valores para o mosto controle, antes da fermentação. No entanto, após o processo fermentativo, observaram-se maiores $(\mathrm{p}<0,05)$ valores de $b^{*}$ para os hidroméis com erva-mate, revelando transformações químicas nos compostos de cor destas amostras. De acordo com Tijskens et al. (2001), o pH exerce grande influência na coloração de alimentos que apresentam o pigmento clorofila. Estes autores sugerem que o íon $\mathrm{Mg}^{2+}$, presente na estrutura da clorofila, pode ser facilmente removido por reação com ácidos fracos e aquecimento, resultando na feofitina, que apresenta coloração tendendo ao marrom oliva. Assim, essas reações bioquímicas podem ter influenciado os resultados do parâmetro b* dos hidroméis HF e HQ.

\subsection{Avaliação do teor de polifenóis totais e da capacidade antioxidante in vitro}

Os resultados dos compostos fenólicos totais (CFT) e da atividade antioxidante para as amostras de mosto e de hidromel, estão apresentados na tabela 2 . 
Tabela 2 - Teor de compostos fenólicos totais (mg EAG/L) e da capacidade antioxidante ( $\mu$ m Trolox/L) pelos métodos ABTS e FRAP das amostras de mosto e de hidromel: controle (MC e HC), com erva-mate com extração à frio (MF e HF) e com ervamate com extração à quente (MQ e HQ).

\begin{tabular}{|c|c|c|c|}
\hline Tratamentos & CFT & ABTS & FRAP \\
\hline $\mathrm{MC}$ & $107,52 \pm 3,70^{\mathrm{aA}}$ & $140,80 \pm 1,95^{\mathrm{aA}}$ & $470,03 \pm 44,21^{\mathrm{aA}}$ \\
\hline MF & $919,09 \pm 31,82^{\mathrm{bA}}$ & $2896,85 \pm 46,26^{\mathrm{bA}}$ & $5244,40 \pm 522,90^{\mathrm{bA}}$ \\
\hline MQ & $1002,42 \pm 64,50^{\mathrm{bA}}$ & $3257,96 \pm 37,82^{\mathrm{cA}}$ & $6042,78 \pm 266,00^{\mathrm{cA}}$ \\
\hline $\mathrm{HC}$ & $156,30 \pm 1,84^{\mathrm{aA}}$ & $222,46 \pm 1,16^{\mathrm{aB}}$ & $1885,73 \pm 272,46^{\mathrm{aB}}$ \\
\hline $\mathrm{HF}$ & $526,67 \pm 11,44^{\mathrm{bB}}$ & $2478,98 \pm 35,17^{\mathrm{bB}}$ & $3138,55 \pm 70,14^{\mathrm{bB}}$ \\
\hline HQ & $597,88 \pm 35,31^{\mathrm{bB}}$ & $2437,31 \pm 163,07^{\mathrm{bB}}$ & $3222,37 \pm 89,47^{\mathrm{bB}}$ \\
\hline
\end{tabular}

${ }^{a-c}$ Diferentes letras minúsculas sobrescritas na mesma coluna indicam diferenças significativas $(\mathrm{p}<0,05)$ entre as amostras para a mesma categoria de amostra (mosto ou hidromel). ${ }^{\text {A-B }}$ Diferentes letras maiúsculas sobrescritas na mesma coluna indicam diferenças significativas $(\mathrm{p}<0,05)$ entre os tratamentos para a mesma amostra. Fonte: Autores $(2021)$.

Os resultados dos compostos fenólicos totais dos mostos demonstram que a amostra controle apresentou menores valores $(\mathrm{p}<0,05)$ em relação aos mostos adicionados de erva-mate. Perfil semelhante também foi observado nos hidroméis. No entanto, após a fermentação observou-se uma redução $(\mathrm{p}<0,05)$ nos teores de CFT das amostras HF e HQ em comparação aos respectivos mostos (MF e MQ), mas sem diferenças ( $p$ > 0,05) entre si, indicando que o tipo de extração realizada não interferiu na quantificação desses compostos. Apesar dessa diminuição, os hidroméis contendo erva-mate apresentaram uma quantidade mais expressiva $(\mathrm{p}<0,05)$ de compostos fenólicos totais em comparação ao hidromel controle. Ribeiro et al. (2019), ao investigarem o potencial de biossorção de compostos fenólicos de erva-mate na biomassa de S. cerevisiae, verificaram uma queda significativa dos CFT do extrato bruto de erva-mate após a biossorção pelas leveduras. Segundo estes autores, o composto fenólico com maior retenção nas células de S. cerevisiae foi o ácido clorogênico, principal composto fenólico da erva-mate. Assim, sugere-se que as leveduras empregadas para a fermentação dos hidroméis podem ter retido em suas células uma parte dos compostos fenólicos dos extratos de erva-mate.

Os resultados para atividade antioxidante indicaram que a adição de erva-mate nos mostos influenciou positivamente $(\mathrm{p}<0,05)$ este parâmetro, com resultados mais expressivos $(\mathrm{p}<0,05)$ para a amostra contendo o extrato de erva-mate com extração à quente (MQ). Ribeiro et al. (2019) ao avaliarem o extrato da erva-mate obtido à quente, observaram resultados semelhante aos obtidos neste estudo, tanto para o método ABTS, quanto FRAP. Da mesma forma, Rodrigues et al. (2015) também obtiveram resultados próximos aos deste estudo em extratos de erva-mate obtidos à frio, ao testarem o método ABTS. Após a fermentação, os resultados expressaram um aumento $(\mathrm{p}<0,05)$ da atividade antioxidante para o hidromel controle e uma diminuição $(\mathrm{p}<0,05)$ para os hidroméis adicionados de erva-mate. No entanto, mesmo ocorrendo uma diminuição da atividade antioxidante após o processo fermentativo, as amostras de hidromel HF e HQ mostraram valores bem superiores ( $\mathrm{p}<0,05)$ em comparação ao hidromel controle. Além disso, os resultados dos hidroméis com erva-mate (HF e HQ) também foram superiores aos observados por Kawa-Rygielska et al. (2019) em hidroméis adicionados de sementes de uva (ABTS - 1180,0 $\mu \mathrm{m} / \mathrm{L}$ e FRAP - $1170 \mu \mathrm{m} / \mathrm{L}$ ), revelando o potencial antioxidante da erva-mate em bebidas fermentadas. 


\subsection{Análise dos componentes principais}

Através dos resultados obtidos neste estudo, foi possível observar que os hidroméis foram influenciados pelos diferentes tratamentos empregados. Com isso, realizou-se a análise de componentes principais (ACP) a fim de destacar as principais diferenças das três amostras de hidroméis (HC, HF e HQ). A ACP (Figura 3) foi realizada com base nos parâmetros físico-químicos (sólidos solúveis totais, $\mathrm{pH}$, acidez total e teor alcoólico), parâmetros de cor ( $\mathrm{L}^{*}, \mathrm{a}^{*}$ e b*), teor de compostos fenólicos totais e capacidade antioxidante (ABTS e FRAP).

Figura 3 - Análise dos componentes principais das amostras de hidromel: controle (HC), com erva-mate com extração à frio (HF) e com erva-mate com extração à quente (HQ).

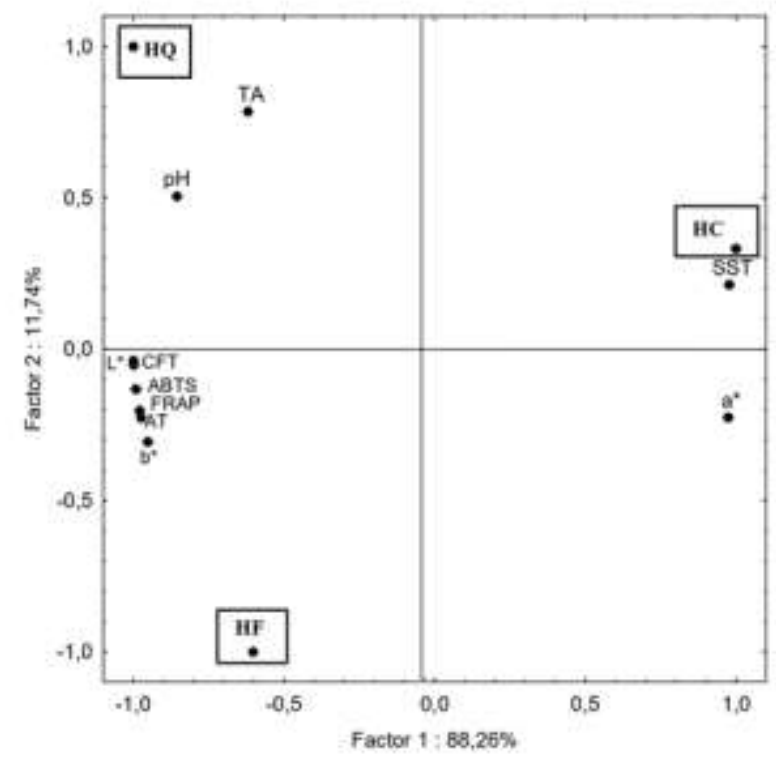

Fonte: Autores (2021). AT: Acidez total; CFT: Compostos fenólicos totais; SST: sólidos solúveis totais; TA: teor alcoólico.

A ACP foi constituída de dois componentes principais (PC1 e PC2) que explicaram 100\% da variabilidade dos dados, onde a PC1 explicou em grande parte a variabilidade dos dados $(88,26 \%)$ e o PC2 explicou 11,74\% da dispersão total. Por meio da ACP, pode-se observar que houve uma separação clara entre as três amostras (HC, HF e HQ), ressaltando que o tratamento realizado durante a elaboração dos hidroméis, consequentemente a adição de erva-mate em pó aos mostos influenciou as características dos hidroméis elaborados.

A PC1 estava relacionada em especial com os compostos fenólicos totais (CFT), a capacidade antioxidante in vitro (ABTS e FRAP), os parâmetros de cor ( $\mathrm{L}^{*}$ e $\mathrm{b}^{*}$ ) e os parâmetros físico-químicos (pH, acidez total e o teor alcoólico), sendo que neste componente houve uma separação clara entre as amostras HF e HQ, sendo que a maioria dos compostos teve associação com a amostra HF, em especial os compostos fenólicos totais e a capacidade antioxidante in vitro. De acordo com PC2, a amostra controle apresentou associação apenas com o teor de sólidos solúveis totais (SST), sendo que o parâmetro de cor a* não apresentou associação com nenhuma das amostras avaliadas.

\section{Considerações finais}

A realização deste trabalho demonstrou que a elaboração de hidromel com adição de erva-mate em pó pode ser uma alternativa viável para produção de um fermentado alcoólico com elevado potencial bioativo. Foi possível notar que a adição 
de erva-mate aumentou o teor de compostos fenólicos totais das bebidas e potencializou a sua atividade antioxidante, sendo que através da análise dos componentes principais pode-se observar que a amostra de extração à frio apresentou associação com os compostos fenólicos totais e a capacidade antioxidante in vitro por ambos os métodos avaliados. Este foi um estudo pioneiro empregando diferentes extratos de erva-mate para produção de hidromel, que revelou resultados promissores. Entretanto, mais estudos são necessários para elucidar a composição fenólica desses hidroméis, bem como a bioacessibilidade dos compostos bioativos no organismo humano, além das propriedades sensoriais das bebidas.

\section{Agradecimentos}

Os autores agradecem ao CNPq (Conselho Nacional de Desenvolvimento Científico e Tecnológico), CAPES (Coordenação de Aperfeiçoamento de Pessoal de Nível Superior) e à FAPESC (Fundação de Amparo à Pesquisa do Estado de Santa Catarina).

\section{Referências}

Akalin, H., Bayram, M., \& Ertan, R. (2017). Determination of some individual phenolic compounds and antioxidant capacity of mead produced from different types of honey. Institute of Brewing \& Distilling, September 2016. https://doi.org/10.1002/jib.396

Andrés-Bello, A., Barreto-Palacios, V., Gárcia-Segovia, P., Mir-Bel, J., \& Martínez-Monzó, J. (2013). Effect of pH on Color and Texture of Food Products. Food Engineering Rewies, 5, 158-170. https://doi.org/10.1007/s12393-013-9067-2

Benzie, I. F. F., \& Strain, J. J. (1996). The Ferric Reducing Ability of Plasma (FRAP) as a Measure of "Antioxidant Power": The FRAP Assay. Analytical Biochemistry, 239(1), 70-76. https://doi.org/10.1006/abio.1996.0292

Boaventura, B. C. B., Silva, E. L. da, Liu, R. H., Prudêncio, E. S., Pietro, P. F. Di, Becker, A. M., \& Amboni, R. D. de M. C. (2015). Effect of yerba mate (Ilex paraguariensis A. St. Hil.) infusion obtained by freeze concentration technology on antioxidant status of healthy individuals. LWT - Food Science and Technology, 62, 948-954. https://doi.org/10.1016/j.lwt.2015.02.028

Chen, C., Wu, Y., Lo, D., \& Wu, M. (2013). Physicochemical property changes during the fermentation of longan (Dimocarpus longan) mead and its aroma composition using multiple yeast inoculations. Institute of Brewing \& Distilling, 119, 303-308. https://doi.org/10.1002/jib.95

Correa, V. G., Gonçalves, G. A., Sá-nakanishi, A. B. De, Ferreira, I. C. F. R., Barros, L., Dias, M. I., Koehnlein, E. A., Souza, C. G. M. De, Bracht, A., \& Peralta, R. M. (2017). Effects of in vitro digestion and in vitro colonic fermentation on stability and functional properties of yerba mate (Ilex paraguariensis A. St Hil.) beverages. Food Chemistry, 237, 453-460. https://doi.org/10.1016/j.foodchem.2017.05.125

Gomes, T., Barradas, C., Dias, T., Verdial, J., Sá, J., Ramalhosa, E., \& Estevinho, L. M. (2013). Optimization of mead production using Response Surface Methodology. Food and Chemical Toxicology, 59, 680-686. https://doi.org/10.1016/j.fct.2013.06.034

IAL, Adolfo Lutz Institute (2008). Analytical Standards of the Adolfo Lutz Institute: Physicochemical Methods for Food Analysis. In IV Edição, $1^{\circ}$ Edição digital (4th ed.). Adolfo Lutz Institute.

Iglesias, A., Pascoal, A., Choupina, A. B., Carvalho, C. A., Feás, X., \& Estevinho, L. M. (2014). Developments in the fermentation process and quality improvement strategies for mead production. Molecules, 19(8), 12577-12590. https://doi.org/10.3390/molecules190812577

Kawa-Rygielska, J., Adamenko, K., Kucharska, A. Z., \& Szatkowska, K. (2019). Fruit and herbal meads - Chemical composition and antioxidant properties. Food Chemistry, 283(July 2018), 19-27. https://doi.org/10.1016/j.foodchem.2019.01.040

Lin, Y., Zhang, W., Li, C., Sakakibara, K., Tanaka, S., \& Kong, H. (2012). Factors affecting ethanol fermentation using Saccharomyces cerevisiae BY4742. Biomass and Bioenergy, 47, 395-401. https://doi.org/10.1016/j.biombioe.2012.09.019

Mendes-Ferreira, A., Cosme, F., Barbosa, C., Falco, V., Inês, A., \& Mendes-faia, A. (2010). International Journal of Food Microbiology Optimization of honey-must preparation and alcoholic fermentation by Saccharomyces cerevisiae for mead production. International Journal of Food Microbiology, 144, 193198. https://doi.org/10.1016/j.ijfoodmicro.2010.09.016

OIV, Organisation of Vine and Wine - (2012). Compendium of international methods of wine and must analysis. In International Organisation of Vine and Wine (2012th ed.). International Organisation of Vine and Wine.

Pereira, A. P., Dias, T., Andrade, J., Ramalhosa, E., \& Estevinho, L. M. (2009). Mead production: Selection and characterization assays of Saccharomyces cerevisiae strains. Food and Chemical Toxicology, 47(8), 2057-2063. https://doi.org/10.1016/j.fct.2009.05.028

Pereira, A. S., Shitsuka, D. M., Parreira, F. J. \& Shitsuka, R. (2018). Metodologia da pesquisa científica. [e-book]. Santa Maria. Ed. UAB/NTE/UFSM. Disponível em: https://repositorio.ufsm.br/bitstream/handle/1/15824/Lic_Computacao_Metodologia-Pesquisa-Cientifica.pdf?sequence=1

Re, R., Pellegrini, N., Proteggente, A., Pannala, A., Yang, M., \& Rice-Evans, C. (1999). Antioxidant activity applying an improved ABTS radical cation decolorization assay. Free Radical Biology \& Medicine, 26(9/10), 1231-1237. 
Ribeiro, V. R., Maciel, G. M., Fachi, M. M., Pontarolo, R., Fernandes, I. de A. A., Stafussa, A. P., \& Haminiuk, C. W. I. (2019). Improvement of phenolic compound bioaccessibility from yerba mate (Ilex paraguariensis) extracts after biosorption on Saccharomyces cerevisiae. Food Research International, 126, 108623. https://doi.org/10.1016/j.foodres.2019.108623

Rodrigues, V. D. C., Da Silva, M. V., Dos Santos, A. R., Zielinski, A. A. F., \& Haminiuk, C. W. I. (2015). Evaluation of hot and cold extraction of bioactive compounds in teas. International Journal of Food Science and Technology, 50(9), 2038-2045. https://doi.org/10.1111/ijfs.12858

Roldán, A., Van Muiswinkel, G. C. J., Lasanta, C., Palacios, V., \& Caro, I. (2011). Influence of pollen addition on mead elaboration: Physicochemical and sensory characteristics. Food Chemistry, 126(2), 574-582. https://doi.org/10.1016/j.foodchem.2010.11.045

Santos, L. P., Caon, T., Battisti, M. A., Silva, C. H. B., Simões, C. M. O., Reginatto, F. H., \& Campos, A. M. de. (2017). Antioxidant polymeric nanoparticles containing standardized extract of Ilex paraguariensis A. St.-Hil. for topical use. Industrial Crops \& Products, 108, 738-747. https://doi.org/10.1016/j.indcrop.2017.07.035

Saxena, S., Gautam, S., \& Sharma, A. (2010). Physical, biochemical and antioxidant properties of some Indian honeys. Food Chemistry, 118(2), 391-397. https://doi.org/10.1016/j.foodchem.2009.05.001

Schmalko, M. E., Scipioni, P. G., \& Ferreyra, D. J. (2005). Effect of water activity and temperature in color and chlorophylls changes in yerba mate leaves. In International Journal of Food Properties (Vol. 8, Issue 2, pp. 313-322). https://doi.org/10.1081/JFP-200060250

Singleton, V. L., \& Rossi, J. A. (1965). Colorimetry of Total Phenolics with Phosphomolybdic-Phosphotungstic Acid Reagents. American Journal of Enology and Viticulture, 16(3), 144 LP - 158. http://www.ajevonline.org/content/16/3/144.abstract

Sroka, P., \& Tuszyński, T. (2007). Changes in organic acid contents during mead wort fermentation. Food Chemistry, 104(3), 1250-1257. https://doi.org/10.1016/j.foodchem.2007.01.046

Starowicz, M., \& Granvogl, M. (2020). Trends in food science \& technology an overview of mead production and the physicochemical, toxicological, and sensory characteristics of mead with a special emphasis on flavor. Trends in Food Science and Technology, 106(March), 402-416. https://doi.org/10.1016/j.tifs.2020.09.006

Thea, A. E., Ferreira, D., Brumovsky, L. A., \& Schmalko, M. E. (2016). Polycyclic aromatic hydrocarbons (PAHs) in yerba maté (Ilex paraguariensis St. Hil) traditional infusions (mate and tereré). Food Control, 60, 215-220. https://doi.org/10.1016/j.foodcont.2015.07.046

Tijskens, L. M. M., Barringer, S. A., \& Biekman, E. S. A. (2001). Modelling the effect of pH on the colour degradation of blanched broccoli. Innovative Food Science and Emerging Technologies, 2(4), 315-322. https://doi.org/10.1016/S1466-8564(01)00048-0 